\title{
Potencial Impacto das Mudanças Climáticas no Zoneamento do Pinus no Espírito Santo
}

\author{
Laís Thomazini Oliveira, José Eduardo Macedo Pezzopane, Roberto Avelino Cecílio
}

${ }^{1}$ Departamento de Engenharia Florestal, Universidade Federal do Espírito Santo - UFES

\begin{abstract}
Resumo
A produção do Pinus no Espírito Santo tornou-se viável devido ao desenvolvimento da tecnologia de utilização de sua madeira nas indústrias de laminados e serrarias. Uma vez que as mudanças climáticas globais podem alterar o cenário silvicultural brasileiro, o objetivo do trabalho foi avaliar seus possíveis impactos sobre o zoneamento climático das espécies de Pinus no Estado. Utilizaram-se dados climáticos atuais de 110 estações e dados climáticos futuros obtidos com base no modelo HadCM3 para a realização do balanço hídrico atual e futuro, necessário ao zoneamento. Verificou-se a existência de $100 \%$ de áreas inaptas na década de 2050 para duas espécies e para todas as espécies na década de 2080.
\end{abstract}

Palavras-chave: aquecimento global, HadCM3, balanço hídrico.

\section{The Potential Impact of Climate Change in the Zoning of Pine Production in the State of Espirito Santo, Brazil}

\begin{abstract}
The production of Pinus in the State of Espirito Santo, Brazil has become feasible due to the development of technology to be used in its timber and lumber laminate industry. Since global climate change may alter the Brazilian forestry scenario, the objective of this paper is to assess its potential impact on the climatic zoning of the pine species in the state. Climate data of 110 stations and future climate data were obtained through HadCM3 model, which was used to calculate the current and future water balance necessary to the zoning. Results showed that $100 \%$ of areas will be unsuitable for two of the species in the 2050s and for all species in the 2080s.
\end{abstract}

Keywords: global warming, HadCM3, water balance.

\section{INTRODUÇÃO}

De acordo com o levantamento coordenado por Bull et al. (1998), os plantios industriais de Pinus no mundo totalizam 18.319 .000 ha, sendo que as maiores áreas cultivadas localizam-se na China, Brasil, Chile e Nova Zelândia.

As espécies do gênero Pinus vêm sendo plantadas em escala comercial no Brasil há mais de 30 anos, ocupando área de cerca de 1,8 milhões de hectares (Revista..., 2008). No Estado do Espírito Santo, as espécies que mais se adaptaram às condições climáticas foram o Pinus elliottii var. elliottii, Pinus caribaea var. hondurensis, Pinus taeda e Pinus oocarpa, porém muitas delas ainda não alcançam sua produtividade potencial em determinados locais 
devido à influência de fatores térmicos e hídricos que limitam seu desenvolvimento.

O cultivo do Pinus é considerado de alto potencial econômico devido a sua grande versatilidade em crescer e produzir madeira nos mais variados tipos de ambiente, bem como a multiplicidade de usos de sua madeira, possibilitando a geração desse recurso natural em todo o território nacional (Empresa..., 2009).

Com o desenvolvimento das indústrias nos setores de papel e celulose, serrados e painéis de madeira, existe hoje grande demanda de madeira superando a atual produção, o que leva à necessidade de expandir o reflorestamento para suprir a demanda existente no País (Castro, 2008). Porém a implantação aleatória de povoamentos florestais e a falta de informações sobre o cultivo de Pinus no Estado tornam os custos para implantação onerosos e, muitas vezes, não se consegue obter a produtividade esperada. Mediante tal fato, é importante a escolha de áreas adequadas para a implantação de cada uma das espécies, de acordo com suas exigências climáticas, por meio de zoneamentos climáticos.

A elaboração de estudos de zoneamento de aptidão climática de culturas consiste em importante ferramenta para o desenvolvimento sustentável da produção vegetal, pois servem de base para a proposta de práticas de manejo agrossilvicultural mais adequadas, para a prevenção e controle das doenças em plantas, ou para a potencialização da produção agrossilvicultural. Segundo Castro (2008), os mapas gerados pelo zoneamento agroclimático definem as áreas com potenciais econômicos para a introdução da espécie em uma determinada região geográfica, servindo como base para pequenos, médios e grandes produtores que queiram investir na cultura, podendo aumentar assim a eficiência e os lucros da atividade.

Dentretodasasatividades econômicas, a produção vegetal é a que apresenta maior dependência das condições ambientais, especialmente as climáticas. Assim, estima-se que qualquer mudança no clima possa afetar o zoneamento agrícola, a produtividade das culturas e as técnicas de manejo, alterando o atual cenário da agricultura brasileira, em cada região, com sérias consequências econômicas, sociais e ambientais (Environmental..., 1989).
Considerando-se os prognósticos de aumento das temperaturas globais, pode-se admitir que as regiões climaticamente limítrofes àquelas de delimitação de cultivo adequado de plantas agrícolas se tornarão desfavoráveis ao desenvolvimento vegetal (Assad et al., 2004). Ainda existem poucos trabalhos relacionando os efeitos das mudanças climáticas com a agrossilvicultura, destacando-se apenas os de Siqueira et al. (1994) e de Assad et al. (2004), não havendo, até o momento, estudos mais consistentes voltados para espécies florestais.

Assim sendo, a previsão do comportamento do zoneamento do Pinus face às mudanças climáticas globais torna-se instrumento de suma importância para a agrossilvicultura de modo a considerar a otimização dos investimentos nesse cultivo.

Do exposto, o presente trabalho teve como objetivo verificar o impacto que as mudanças climáticas globais poderão exercer sobre o zoneamento agroclimático das espécies Pinus elliottii var. elliottii, Pinus caribaea var. hondurensis, Pinus taeda e Pinus oocarpa no Estado do Espírito Santo.

\section{MATERIAL E MÉTODOS}

A área contemplada no presente estudo é o Estado do Espírito Santo, com $46.184 \mathrm{~km}^{2}$ de superfície, situada entre os meridianos $39^{\circ} 38^{\prime}$ e $41^{\circ} 50^{\prime}$ de longitude oeste e entre os paralelos $17^{\circ} 52^{\prime}$ e $21^{\circ} 19^{\prime}$ de latitude sul. Conforme o sistema de classificação de Köppen, a região enquadra-se nos grupos climáticos A e C (úmidos), sendo encontrados os subtipos climáticos Aw, Am, Cf e Cw, e também as variações Cfa, Cfb, Cwa e Cwb (Siqueira et al., 2004).

Calcularam-se, para 110 estações pluviométricas localizadas no Espírito Santo e nas regiões circunvizinhas, os balanços hídricos climatológicos a partir do método preconizado por Thornthwaite \& Mather (1955) para valores médios mensais de temperatura do ar e precipitação, adotando-se $300 \mathrm{~mm}$ como capacidade máxima de retenção de água pelo solo (CAD). As precipitações médias mensais de cada estação foram obtidas com o uso de séries de dados de chuva com um mínimo de 20 anos de registros, obtidas por meio do sistema HidroWeb da Agência Nacional de Águas (http \l:hidroweb. 
ana.gov.br) e cedidas pelo Instituto Capixaba de Pesquisa, Assistência Técnica e Extensão Rural (INCAPER). Para as estações que não dispunham de dados de temperatura média mensal, estes valores foram estimados por meio das equações proposta por Castro (2008), Sediyama \& Melo Júnior (1998), Medeiros et al. (2005) e Alfonsi et al. (2007) para o Espírito Santo, Minas Gerais, Bahia e Rio de Janeiro, respectivamente. A evapotranspiração potencial foi estimada pelo método de Thornthwaite (1948), baseado apenas na temperatura média do ar e nas coordenadas geográficas do local. Estes dados assim obtidos consistiram nos valores médios atuais de elementos do clima e do balanço hídrico.

Os dados climáticos futuros foram obtidos do IPCC (Intergovernmental..., 2007), disponível no sítio http://www.ipcc.ch, com definição de área de estudo para o Brasil. Utilizaram-se dois cenários futuros - A2 e B2 -, projetados para as décadas de 2020 (entre 2010 e 2039), 2050 (entre 2040 e 2069) e 2080 (entre 2070 e 2099): A2 é o cenário que descreve um mundo futuro muito heterogêneo no qual a regionalização é dominante; e B2 é o cenário que descreve um mundo no qual a ênfase está em soluções locais para a sustentabilidade econômica, social e ambiental. O modelo de previsão do clima adotado foi o HadCM3, elaborado por Hadley Centre for Climate Prediction and Research, do qual se obtiveram os dados relativos aos desvios previstos para a temperatura do ar e a precipitação, em escalas mensais, nos cenários futuros em cada região do globo, que foram somados aos dados climáticos atuais. De posse dos valores de temperatura do ar e precipitações mensais do futuro, mais uma vez foram calculados os balanços hídricos climatológicos para cada cenário, em cada período de tempo analisado. Desta forma, foram caracterizadas seis condições climáticas futuras, relativas a dois cenários e três períodos de tempo em cada cenário.

Para zonear áreas potencialmente aptas ao cultivo do Pinus no Estado do Espírito Santo, foram utilizados os dados referentes aos seguintes elementos climáticos do balanço hídrico climatológico: temperatura média anual (Ta) e o déficit hídrico anual $(\mathrm{Da})$. As faixas de aptidão térmica e hídrica de acordo com Carpanezzi et al. (1986), Golfari et al.
(1978) e Castro et al. (2010b), estão ilustradas nas Tabelas 1 e 2.

As áreas foram classificadas de acordo com as necessidades de cada espécie em duas classes de aptidão climática, como seguem:

- Apta: condições térmicas e hídricas da área apresentam-se favoráveis para o bom desenvolvimento e produção da espécie em escala comercial.

- Inapta: quando as características normais do clima não se apresentam adequadas à exploração econômica da espécie, devido a limitações severas dos fatores hídricos ou térmicos, ou ambos com marcante repercussão em sua produção, exigindo para que sejam corrigidas práticas agrícolas dispendiosas.

As informações a respeito dos elementos climáticos foram manipuladas por meio dos softwares IDRISI (da Universidade de Clark, EUA) e SPRING (do Instituto nacional de Pesquisas Espaciais - INPE, Brasil) de modo a formar um banco de dados que conteve diversas imagens digitais especializadas, em formato matricial (raster), relativas a cada elemento

Tabela 1. Faixa de aptidão térmica para as espécies de Pinus.

Table 1. Thermal range suitability for the pine species.

\begin{tabular}{ll}
\multirow{1}{*}{ Cultura } & \multicolumn{1}{c}{ Regiões } \\
\cline { 2 - 2 } & $\begin{array}{c}\text { Apta inapta } \\
\text { Temperatura média anual (Ta) }\end{array}$ \\
\hline $\begin{array}{l}\text { Pinus elliottii var. } \\
\text { elliottii }\end{array}$ & $15 \leq \mathrm{Ta} \leq 24^{\circ} \mathrm{C} 15>\mathrm{Ta}>24^{\circ} \mathrm{C}$ \\
$\begin{array}{l}\text { Pinus caribaea var. } \\
\text { hondurensis }\end{array}$ & $21 \leq \mathrm{Ta} \leq 27^{\circ} \mathrm{C} 21>\mathrm{Ta}>27^{\circ} \mathrm{C}$ \\
$\begin{array}{l}\text { Pinus oocarpa } \\
\text { Pinus taeda }\end{array}$ & $13 \leq \mathrm{Ta} \leq 21^{\circ} \mathrm{C} 13>\mathrm{Ta}>21^{\circ} \mathrm{C}$ \\
\hline
\end{tabular}

Tabela 2. Faixa de aptidão hídrica para as espécies de Pinus.

Table 2. Water range suitability for the pine species.

\begin{tabular}{|lc} 
& \multicolumn{1}{c}{ Regiões } \\
\cline { 2 - 2 } \multicolumn{1}{c}{ Cultura } & Apta inapta \\
\cline { 2 - 2 } & $\begin{array}{c}\text { Deficiência hídrica } \\
\text { anual (Da) }\end{array}$ \\
Pinus elliottii var. elliottii & $\leq 50 \mathrm{~mm}>50 \mathrm{~mm}$ \\
Pinus caribaea var. hondurensis & $\leq 200 \mathrm{~mm}>200 \mathrm{~mm}$ \\
Pinus oocarpa & $\leq 200 \mathrm{~mm}>200 \mathrm{~mm}$ \\
Pinus taeda & $\leq 50 \mathrm{~mm}>50 \mathrm{~mm}$
\end{tabular}


necessário para realização dos zoneamentos propostos.

Para obter o mapa da espacialização da temperatura média anual do ar, aplicaram-se os coeficientes obtidos por meio da Equação 1 (Castro, 2008) a imagens digitais de altitude, latitude e longitude. As imagens de altitude foram obtidas por meio de um modelo digital de elevação (MDE), obtido através de dados de radar SRTM (Shuttle Radar Topography Mission).

$\mathrm{T}=-12,9848-0,0071 \mathrm{ALT}+0,4690 \mathrm{LAT}-1,1761 \mathrm{LONG}(1)$

em que: $\mathrm{T}$ - temperatura média anual em ${ }^{\circ} \mathrm{C}$; LONG - longitude do local em graus decimais (valores positivos); LAT - latitude do local em graus e décimos; e ALT - altitude do local em metros.

O método de interpolação utilizado na espacialização da deficiência hídrica anual foi a krigagem esférica, com efeito pepita igual a 2984,601, patamar igual a 21387,41 e alcance igual a $134,5 \mathrm{~km}$, conforme sugerido por Castro et al. (2010a).

Com base nos mapas temáticos de temperatura média anual do ar e deficiência hídrica, foi feita uma reclassificação de acordo com as exigências climáticas de cada espécie. Assim, obtiveram-se os mapas de zoneamento com a classificação das áreas aptas e inaptas ao desenvolvimento das espécies, seguindo-se os procedimentos ilustrados na Figura 1, adaptados da metodologia proposta por Cecílio et al. (2006).

\section{RESULTADOS E DISCUSSÃO}

Os mapas temáticos do zoneamento climático atual para a implantação das espécies Pinus elliottii var. elliottii, Pinus caribaea var. hondurensis, Pinus oocarpa e Pinus taeda no Espírito Santo, conforme as exigências climáticas de cada espécie, estão ilustrados na Figura 2. A Tabela 3 apresenta o percentual das áreas aptas e inaptas para a implantação do Pinus spp. no cenário atual para o Estado do Espírito Santo, na qual observa-se que o Espírito Santo apresenta áreas com aptidão climática para todas as espécies de Pinus, no cenário atual, de acordo com as bases estabelecidas para este zoneamento.

A espécie Pinus caribaea var. hondurensis apresenta a maior área de aptidão quando comparada com as espécies Pinus elliottii var. elliottii, Pinus oocarpa e Pinus taeda, pois essas três possuem, como exigências climáticas, temperatura mais amena e menor deficiência hídrica.

Conforme ilustra a Figura 2, as áreas com aptidão para o plantio do Pinus elliottii var. elliottii abrangem grande parte do sul do Estado, principalmente aquelas mais elevadas onde a temperatura do ar é mais baixa e a deficiência hídrica pequena. Tal característica é observada, de forma similar, para a espécie Pinus taeda, que pode se desenvolver apenas em uma pequena porção da região serrana do Espírito Santo, por possuir maior altitude, com temperaturas do ar mais amenas.

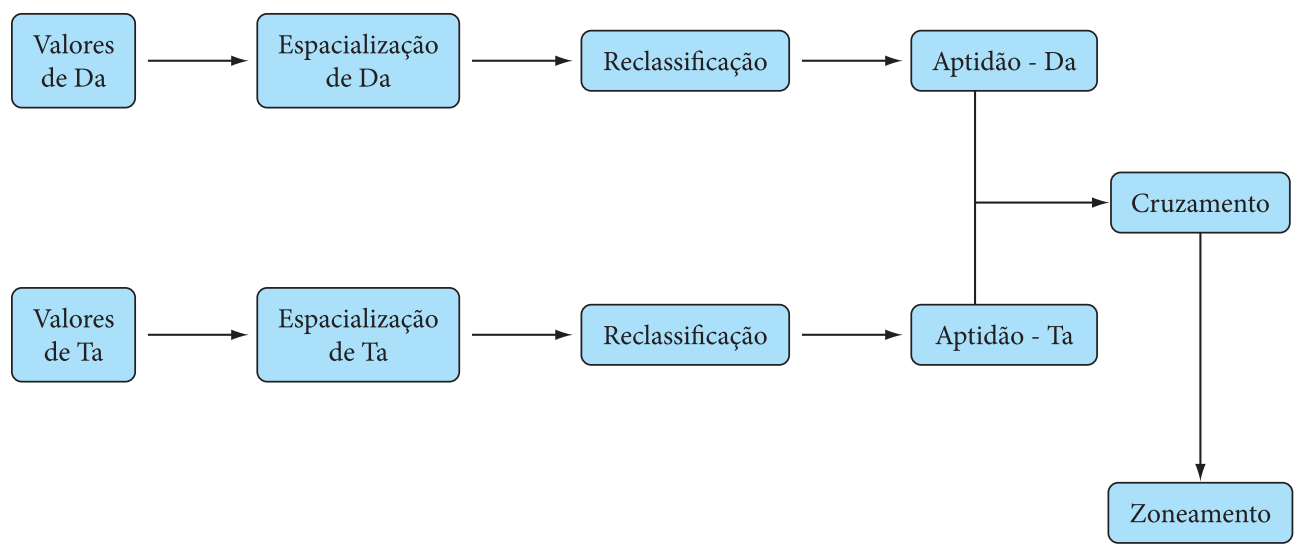

Figura 1. Fluxograma das etapas necessárias para obtenção dos mapas de zoneamento agroclimático da cultura do Pinus no Estado do Espírito Santo.

Figure 1. Flowchart of the steps to obtain maps of Pinus agroclimatic zoning in the State of Espirito Santo. 


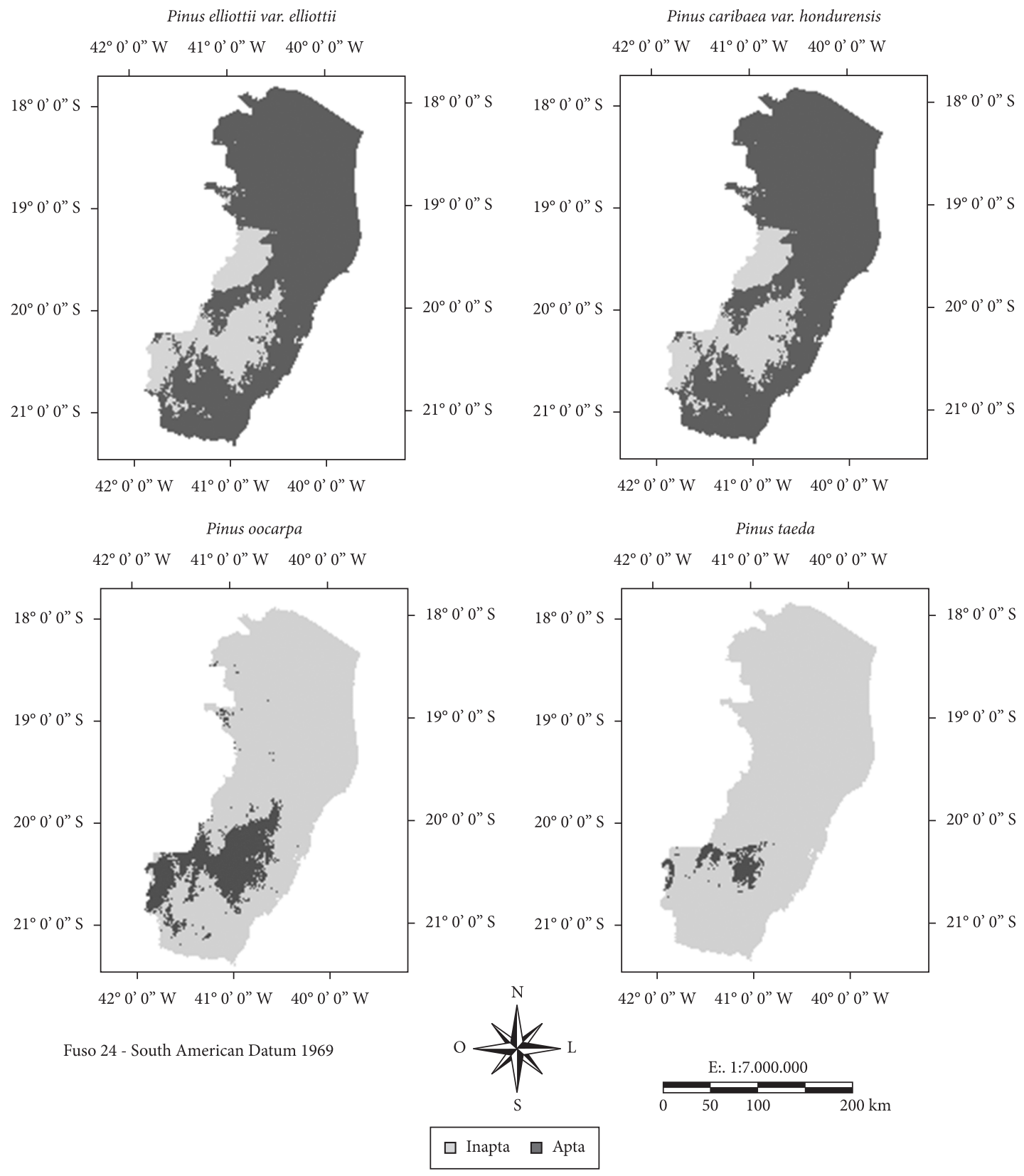

Figura 2. Zoneamento agroclimático para as espécies Pinus elliottii var. elliottii, Pinus caribaea var. hondurensis, Pinus oocarpa e Pinus taeda no cenário atual.

Figure 2. Agroclimatic zoning for the species Pinus elliottii var. elliottii, Pinus caribaea var. hondurensis, Pinus taeda and Pinus oocarpa in the current scenario.

Com relação à espécie Pinus oocarpa, a área climaticamente apta para a implantação dessa espécie no Estado é maior que aquela do Pinus taeda, sendo que as duas espécies apresentam exigências térmicas muito próximas. Com relação à deficiência hídrica, o Pinus oocarpa consegue tolerar até $200 \mathrm{~mm}$, enquanto o Pinus taeda tolera somente até $50 \mathrm{~mm}$ Tal ocorrência explica a maior porcentagem da área apta ao cultivo do Pinus oocarpa.

De acordo com as exigências climáticas do Pinus caribaea var. hondurensis, este pode ser implantado em regiões mais quentes, porém com deficiência 
hídrica menor que $200 \mathrm{~mm}$, sendo essa espécie mais indicada para a implantação na maior parte do Estado, como ilustra a Figura 2. Esta espécie pode ser cultivada por todo o litoral e na maioria dos municípios do norte do Espírito Santo, o que não ocorre para o Pinus elliottii var. elliottii, Pinus oocarpa e Pinus taeda. Remade (Revista..., 2002) coborra tal fato, recomendando a implantação do Pinus caribaea var. hondurensis em quase todo o território do Brasil, incluindo toda a faixa litorânea.

De maneira geral, Castro et al. (2010b) obtiveram resultados semelhantes em seus estudos, com pequenas variações nos percentuais de áreas aptas e inaptas para o Pinus elliottii var. elliottii, Pinus oocarpa e Pinus taeda e maior variação para o Pinus caribaea var. hondurensis com 54,4\% de área apta para o cultivo desta espécie. No entanto, manteve a espécie Pinus caribaea var. hondurensis com maior área apta para o cultivo e o Pinus taeda com menor área para sua implantação.

Tabela 3. Áreas aptas e inaptas para a implantação de Pinus spp. no Estado.

Table 3. Suitable and unsuitable areas for Pinus spp. in the State of Espirito Santo.

\begin{tabular}{lcc|}
\multicolumn{1}{|c}{ Espécies } & $\begin{array}{c}\text { Áreas aptas } \\
\text { (\% do total) }\end{array}$ & $\begin{array}{c}\text { Áreas inaptas } \\
\text { (\% do total) }\end{array}$ \\
\hline $\begin{array}{l}\text { Pinus elliottii var. } \\
\text { elliottii }\end{array}$ & 14,96 & 85,04 \\
\hline $\begin{array}{l}\text { Pinus caribaea var. } \\
\text { hondurensis }\end{array}$ & 78,92 & 21,08 \\
\hline Pinus oocarpa & 15,73 & 84,27 \\
\hline Pinus taeda & 3,52 & 96,48 \\
\hline
\end{tabular}

A Tabela 4 apresenta o percentual das áreas consideradas aptas e inaptas para a implantação do Pinus elliottii var. elliotti, Pinus caribaea var. hondurensis, Pinus oocarpa e Pinus taeda nas décadas de 2020, 2050 e 2080, nos cenários, A2 e B2.

A Figura 3 apresenta os mapas temáticos do zoneamento agroclimático para a implantação da espécie Pinus elliottii var. elliottii, no Espírito Santo, conforme as exigências climáticas da espécie, para as décadas de 2020, 2050 e 2080 nos cenários, A2 e B2.

Conforme observou-se na Figura 2, as áreas com aptidão para o plantio da espécie Pinus elliottii var. elliottii abrangem atualmente uma parte significativa do sul do Estado, principalmente aquelas áreas mais elevadas onde a temperatura do ar é mais baixa e a deficiência hídrica pequena. Como evidenciado na Figura 3, em resposta a mudanças climáticas globais, observa-se potencial redução das áreas de aptidão dessa espécie no decorrer das décadas futuras, comprometendo o desenvolvimento e o estabelecimento dessa espécie no Estado.

O comportamento do Pinus elliottii var. elliottii face às mudanças climáticas é crítico, apresentando sérias restrições ao seu cultivo na maior parte do Estado, pois a faixa de aptidão térmica e hídrica é reduzida pelo aumento da temperatura e redução da precipitação nos cenários futuros. Tal fato torna a implantação desta espécie no Estado incompatível com suas exigências climáticas, resultando em pequenas áreas aptas para o cultivo da espécie na década de 2020, e totalmente inadequada para as próximas décadas, já que esta redução alcança o valor

Tabela 4. Áreas aptas e inaptas para a implantação de Pinus ssp. no Estado, nas décadas de 2020, 2050 e 2080, com seus respectivos cenários.

Table 4. Suitable and unsuitable areas for Pinus spp. in the State of Espirito Santo in the decades of 2020, 2050 and 2080, with their respective scenarios.

\begin{tabular}{|c|c|c|c|c|c|c|c|c|}
\hline \multirow{3}{*}{ Décadas } & \multicolumn{8}{|c|}{ Áreas (\% do total) } \\
\hline & \multicolumn{2}{|c|}{$\begin{array}{c}\text { Pinus elliottii var. } \\
\text { elliottii }\end{array}$} & \multicolumn{2}{|c|}{$\begin{array}{c}\text { Pinus caribaea var. } \\
\text { hondurensis }\end{array}$} & \multicolumn{2}{|c|}{ Pinus oocarpa } & \multicolumn{2}{|c|}{ Pinus taeda } \\
\hline & Aptas & Inaptas & Aptas & Inaptas & Aptas & Inaptas & Aptas & Inaptas \\
\hline 2020 A2 & 4,73 & 95,27 & 60,60 & 39,4 & 9,96 & 90,04 & 0,89 & 99,11 \\
\hline 2020 B2 & 2,01 & 97,99 & 29,71 & 70,29 & 8,63 & 91,37 & 0,40 & 99,6 \\
\hline 2050 A2 & 0,0 & 100,0 & 14,21 & 85,79 & 3,37 & 96,63 & 0,0 & 100,0 \\
\hline 2050 B2 & 0,0 & 100,0 & 17,09 & 82,91 & 4,90 & 95,1 & 0,0 & 100,0 \\
\hline $2080 \mathrm{~A} 2$ & 0,0 & 100,0 & 0,0 & 100,0 & 0,0 & 100,0 & 0,0 & 100,0 \\
\hline 2080 B2 & 0,0 & 100,0 & 5,0 & 95,0 & 1,0 & 99,0 & 0,0 & 100,0 \\
\hline
\end{tabular}


Cenário A2

$42^{\circ} 0^{\prime} 0^{\prime \prime} \mathrm{W} \quad 41^{\circ} 0^{\prime} 0^{\prime \prime} \mathrm{W} \quad 40^{\circ} 0^{\prime} 0^{\prime \prime} \mathrm{W}$

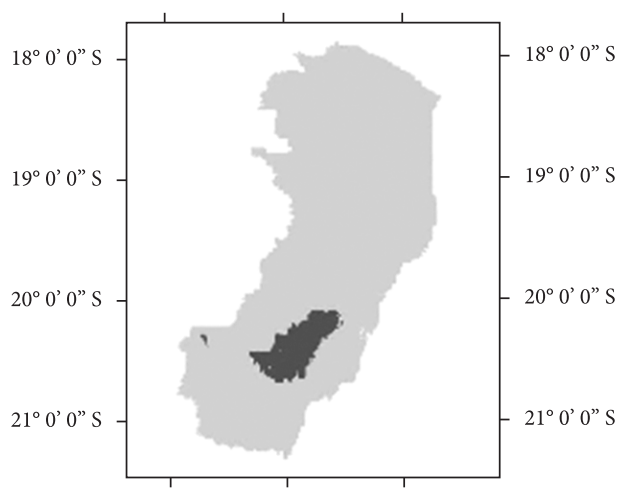

$42^{\circ} 0^{\prime} 0^{\prime \prime} \mathrm{W} 41^{\circ} 0^{\prime} 0^{\prime \prime} \mathrm{W} 40^{\circ} 0$ ' $0^{\prime \prime} \mathrm{W}$

$42^{\circ} 0^{\prime} 0^{\prime \prime} \mathrm{W} 41^{\circ} 0^{\prime} 0$ " W $40^{\circ} 0^{\prime} 0^{\prime \prime} \mathrm{W}$

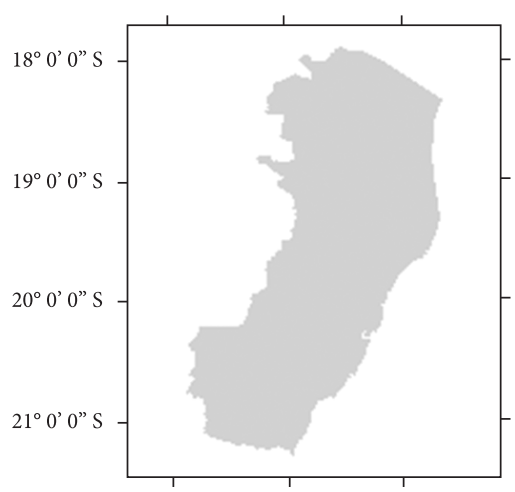

$42^{\circ} 0^{\prime} 0$ " W $41^{\circ} 0^{\prime} 0$ " W $40^{\circ} 0^{\prime} 0^{\prime \prime} \mathrm{W}$

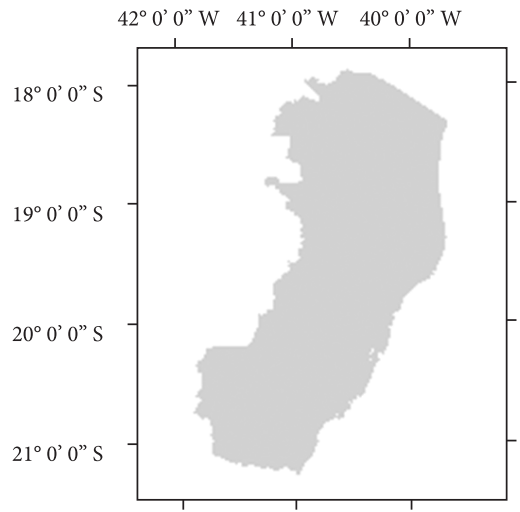

$42^{\circ} 0^{\prime} 0^{\prime \prime} \mathrm{W} \quad 41^{\circ} 0^{\prime} 0^{\prime \prime} \mathrm{W} \quad 40^{\circ} 0^{\prime} 0^{\prime \prime} \mathrm{W}$

Fuso 24 - South American Datum 1969

$18^{\circ} 0^{\prime} 0^{\prime \prime} \mathrm{S}$

$19^{\circ} 0$ '0" S

$20^{\circ} 0$ ' 0" S

$21^{\circ} 0$ ' 0" S

$18^{\circ} 0^{\prime} 0^{\prime \prime} \mathrm{S}$

$19^{\circ} 0^{\prime} 0^{\prime \prime} \mathrm{S}$

$20^{\circ} 0^{\prime} 0$ " S

$21^{\circ} 0$ ' 0" S
Cenário B2

$42^{\circ} 0^{\prime} 0$ " W $41^{\circ} 0^{\prime} 0^{\prime \prime} \mathrm{W} 40^{\circ} 0^{\prime} 0^{\prime \prime} \mathrm{W}$

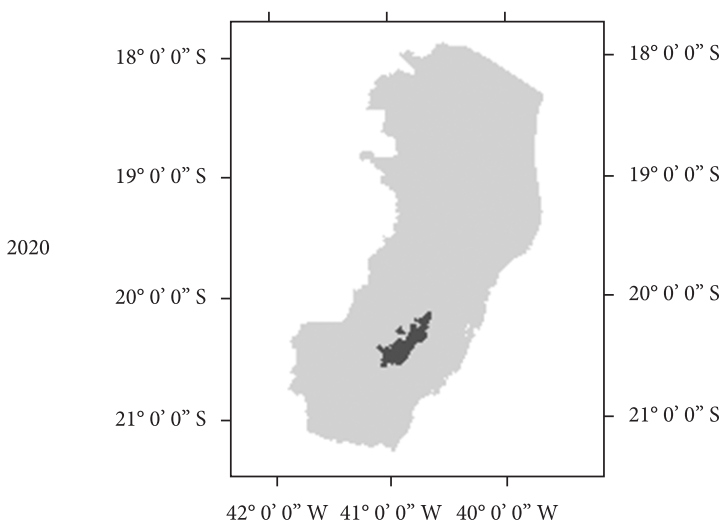

$42^{\circ} 0^{\prime} 0^{\prime \prime} \mathrm{W} \quad 41^{\circ} 0^{\prime} 0^{\prime \prime} \mathrm{W} \quad 40^{\circ} 0^{\prime} 0^{\prime \prime} \mathrm{W}$

2050
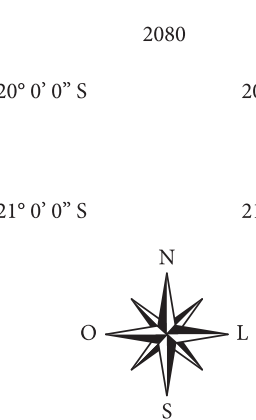

$21^{\circ} 0^{\prime} 0^{\prime \prime} \mathrm{S}$

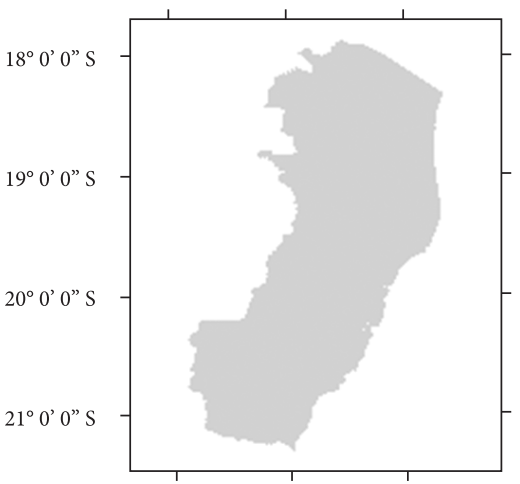

$18^{\circ} 0^{\prime} 0^{\prime \prime} \mathrm{S}$

$19^{\circ} 0$ ' 0" S

$20^{\circ} 0^{\prime} 0^{\prime \prime} \mathrm{S}$

$21^{\circ} 0^{\prime} 0^{\prime \prime} \mathrm{S}$

$42^{\circ} 0^{\prime} 0$ " W $41^{\circ} 0^{\prime} 0$ " W $40^{\circ} 0^{\prime} 0$ " W

$42^{\circ} 0^{\prime} 0$ " W $41^{\circ} 0^{\prime} 0$ " W $40^{\circ} 0^{\prime} 0^{\prime \prime} \mathrm{W}$

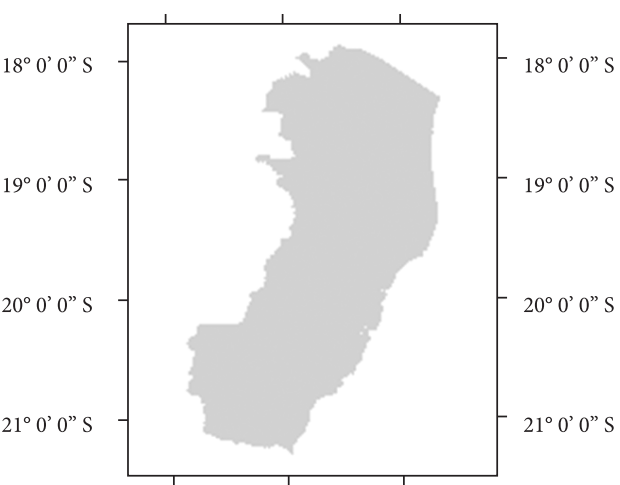

$42^{\circ} 0$ 0 0" W $41^{\circ} 0$ ' 0 " W $40^{\circ} 0^{\prime} 0^{\prime \prime} \mathrm{W}$

E. $1: 7.000 .000$

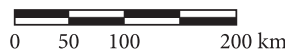

$\square$ Inapta

$\square$ Apta

Figura 3. Zoneamento agroclimático para a espécie Pinus elliottii var. elliottii, nas décadas de 2020, 2050 e 2080, com seus respectivos cenários.

Figure 3. Agroclimatic zoning for Pinus elliottii var. elliottii in the decades of 2020, 2050 and 2080, with their respective scenarios. 
de $100 \%$ a partir da década de 2050 (Tabela 4). Podese observar uma pequena diferença entre os cenários analisados, sendo o cenário A2 menos desfavorável à implantação da espécie.

A Figura 4 apresenta o zoneamento agroclimático para a espécie Pinus caribaea var. hondurensis nas décadas de 2020, 2050 e 2080, nos cenários, A2 e B2. Pode-se observar que o percentual das áreas aptas ao cultivo desta espécie é significativamente maior quando comparado com o das espécies Pinus elliottii var. elliottii, Pinus oocarpa e Pinus taeda (Tabela 4). Apesar das características térmicas e hídricas serem favoráveis ao cultivo dessa espécie no Estado, podese observar a redução expressiva das áreas de aptidão climática com as mudanças climáticas (Figura 4).

Atualmente, as áreas aptas ao cultivo do Pinus caribaea var. hondurensis apresentam um desvio para a região sul e serrana do Estado (Figura 2), regiões estas mais altas, com temperaturas mais amenas e maiores volumes de precipitação no decorrer do ano. O impacto das mudanças climáticas no comportamento desta espécie foi menor quando comparado ao do Pinus elliottii var. elliottii, devido à exigência hídrica desta espécie ser menor e suportar melhor a redução das precipitações e aumento da temperatura ao longo das décadas.

A Figura 5 mostra o zoneamento agroclimático para a espécie Pinus oocarpa, nas décadas de 2020, 2050 e 2080, nos cenários A2 e B2, conforme suas exigências climáticas, nas quais as áreas climaticamente aptas para a implantação dessa espécie no Estado são maiores quando comparadas a do Pinus taeda (Figura 6 e Tabela 4), sendo que as duas espécies apresentam exigências térmicas muito próximas.

O Pinus oocarpa apresenta menores áreas cultiváveis quando comparado ao Pinus caribaea var. hondurensis e maiores valores quando comparados ao Pinus elliottii var. elliottii e Pinus taeda, fato devido à sua exigência hídrica ser a mesma do $P$. caribaea var. hondurensis e exigência térmica próximas às outras espécies.

O resultado da Tabela 4 apresenta este mesmo comportamento quanto aos valores das áreas aptas e inaptas ao cultivo do Pinus oocarpa perante as mudanças climáticas, demonstrando o aumento das áreas impróprias. No entanto, essa espécie apresenta maior tolerância às mudanças climáticas quando comparada com as espécies Pinus elliottii var. elliottii e Pinus taeda. Observa-se que o cenário A2 apresentou melhor comportamento na década de 2020, enquanto o cenário B2 apresentou maiores valores nas décadas de 2050 e 2080, fato observado para a espécie $P$. caribaea var. hondurensis, que confirma o cenário $\mathrm{B} 2$ como o cenário otimista para a análise dos impactos das mudanças climáticas, sendo que, com o passar do tempo, a tendência é o aumento da temperatura comprometendo o estabelecimento de diversas espécies no planeta.

A Figura 6 apresenta o zoneamento agroclimático para a implantação da espécie Pinus taeda, conforme as exigências climáticas da espécie, para as décadas de 2020, 2050 e 2080 nos cenários, A2 e B2.

A Tabela 4 mostra o impacto das mudanças climáticas para a espécie Pinus taeda, com o aumento das áreas impróprias, que alcança o valor de $100 \%$. Essa espécie apresenta os menores valores de áreas aptas para seu cultivo, demonstrando que suas limitações hídricas e térmicas são muito restritas. Tal comportamento pode ser observado para a espécie Pinus elliottii var. elliotti.

O comportamento da espécie Pinus taeda foi o mais afetado face às mudanças climáticas, pois esta apresenta uma restrita faixa de aptidão térmica e não tolera deficiência hídrica maior que $50 \mathrm{~mm}$.

É notável que as espécies com maiores restrições térmicas e hídricas, Pinus elliottii var. elliottii e Pinus taeda tiveram o cenário A2 como o mais promissor para todas as décadas futuras analisadas, para o cultivo no Estado. Entretanto, as espécies menos exigentes climaticamente, Pinus caribaea var. hondurensis e Pinus oocarpa, tiveram melhor comportamento no cenário A2 na década de 2020, enquanto nas décadas de 2050 e 2080 o cenário B2 foi o mais promissor para o desenvolvimentos destas espécies. Fato aliado a este é a deficiência hídrica ser a variável que mais influencia no comportamento das espécies, tanto no zoneamento atual, como nas previsões futuras realizadas para a obtenção de mapas que delimitam as áreas aptas e inaptas ao cultivo do Pinus ssp.

Com relação às previsões futuras, percebese claramente a tendência de grande aumento de áreas inaptas ao cultivo do Pinus ssp. ao longo das décadas, o que se deve ao incremento excessivo da 
Cenário A2

$42^{\circ} 0^{\prime} 0^{\prime \prime} \mathrm{W} 41^{\circ} 0^{\prime} 0^{\prime \prime} \mathrm{W} 40^{\circ} 0^{\prime} 0^{\prime \prime} \mathrm{W}$

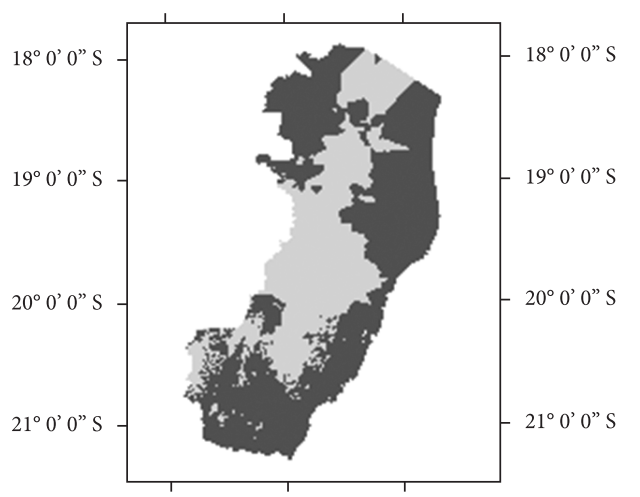

$42^{\circ} 0^{\prime} 0^{\prime \prime} \mathrm{W} 41^{\circ} 0^{\prime} 0^{\prime \prime} \mathrm{W} 40^{\circ} 0^{\prime} 0^{\prime \prime} \mathrm{W}$

$42^{\circ} 0^{\prime} 0^{\prime \prime} \mathrm{W} 41^{\circ} 0^{\prime} 0^{\prime \prime} \mathrm{W} \quad 40^{\circ} 0^{\prime} 0^{\prime \prime} \mathrm{W}$

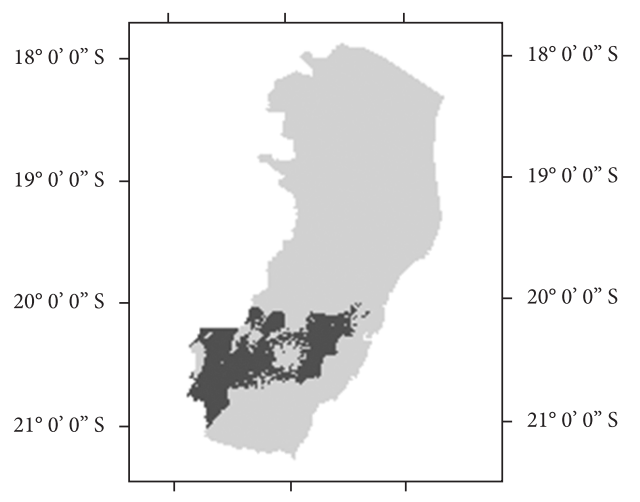

$42^{\circ} 0^{\prime} 0^{\prime \prime} \mathrm{W} 41^{\circ} 0^{\prime} 0^{\prime \prime} \mathrm{W} 40^{\circ} 0^{\prime} 0^{\prime \prime} \mathrm{W}$

$42^{\circ} 0^{\prime} 0^{\prime \prime} \mathrm{W} 41^{\circ} 0^{\prime} 0^{\prime \prime} \mathrm{W} 40^{\circ} 0^{\prime} 0^{\prime \prime} \mathrm{W}$

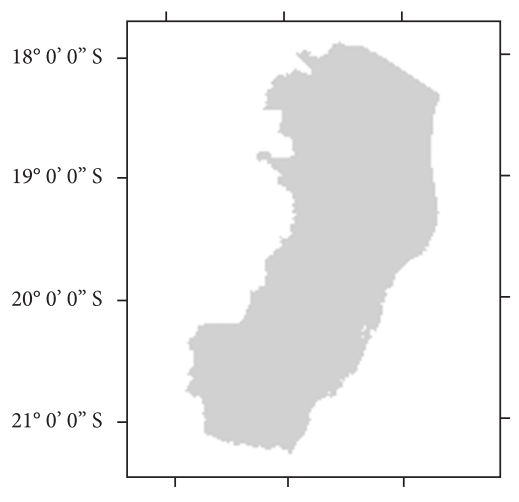

$42^{\circ} 0^{\prime} 0^{\prime \prime} \mathrm{W} 41^{\circ} 0^{\prime} 0^{\prime \prime} \mathrm{W} 40^{\circ} 0^{\prime} 0^{\prime \prime} \mathrm{W}$

Fuso 24 - South American Datum 1969
Cenário $\mathrm{B} 2$

$42^{\circ} 0^{\prime} 0^{\prime \prime} \mathrm{W} \quad 41^{\circ} 0^{\prime} 0^{\prime \prime} \mathrm{W} \quad 40^{\circ} 0^{\prime} 0^{\prime \prime} \mathrm{W}$

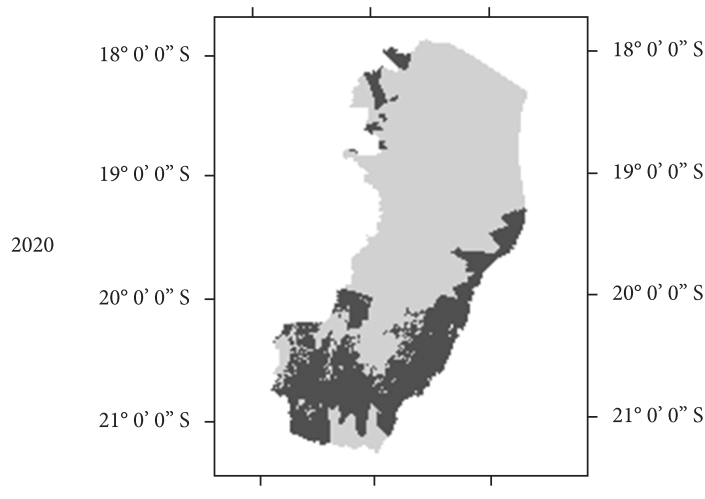

$42^{\circ} 0^{\prime} 0^{\prime \prime} \mathrm{W} 41^{\circ} 0^{\prime} 0^{\prime \prime} \mathrm{W} 40^{\circ} 0^{\prime} 0^{\prime \prime} \mathrm{W}$

$42^{\circ} 0^{\prime} 0^{\prime \prime} \mathrm{W} 41^{\circ} 0^{\prime} 0^{\prime \prime} \mathrm{W} 40^{\circ} 0^{\prime} 0^{\prime \prime} \mathrm{W}$

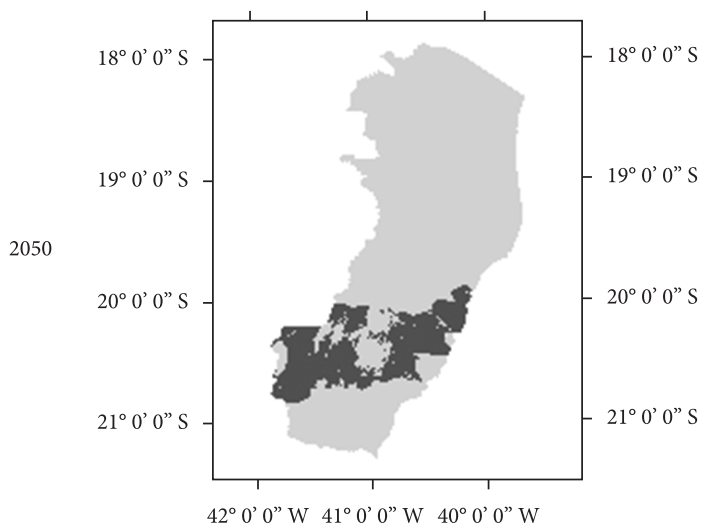

$42^{\circ} 0^{\prime} 0^{\prime \prime} \mathrm{W} 41^{\circ} 0^{\prime} 0^{\prime \prime} \mathrm{W} 40^{\circ} 0^{\prime} 0^{\prime \prime} \mathrm{W}$

$42^{\circ} 0^{\prime} 0^{\prime \prime} \mathrm{W} \quad 41^{\circ} 0^{\prime} 0^{\prime \prime} \mathrm{W} \quad 40^{\circ} 0^{\prime} 0^{\prime \prime} \mathrm{W}$

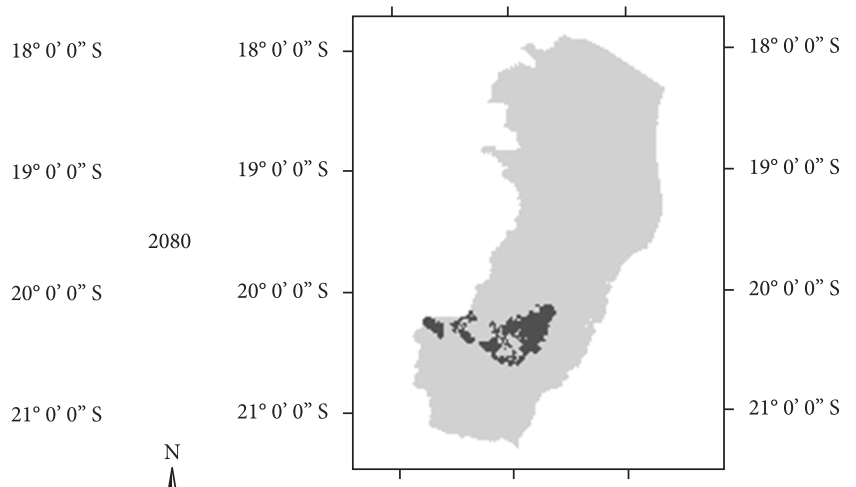

$42^{\circ} 0^{\prime} 0^{\prime \prime} \mathrm{W} 41^{\circ} 0^{\prime} 0^{\prime \prime} \mathrm{W} 40^{\circ} 0^{\prime} 0^{\prime \prime} \mathrm{W}$

E.: 1:7.000.000

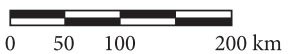

$\square$ Inapta $\square$ Apta

Figura 4. Zoneamento agroclimático para a espécie Pinus caribaea var. hondurensis nas décadas de 2020, 2050 e 2080, com seus respectivos cenários.

Figure 4. Agroclimatic zoning for Pinus caribaea var. hondurensis in the decades of 2020, 2050 and 2080, with their respective scenarios. 


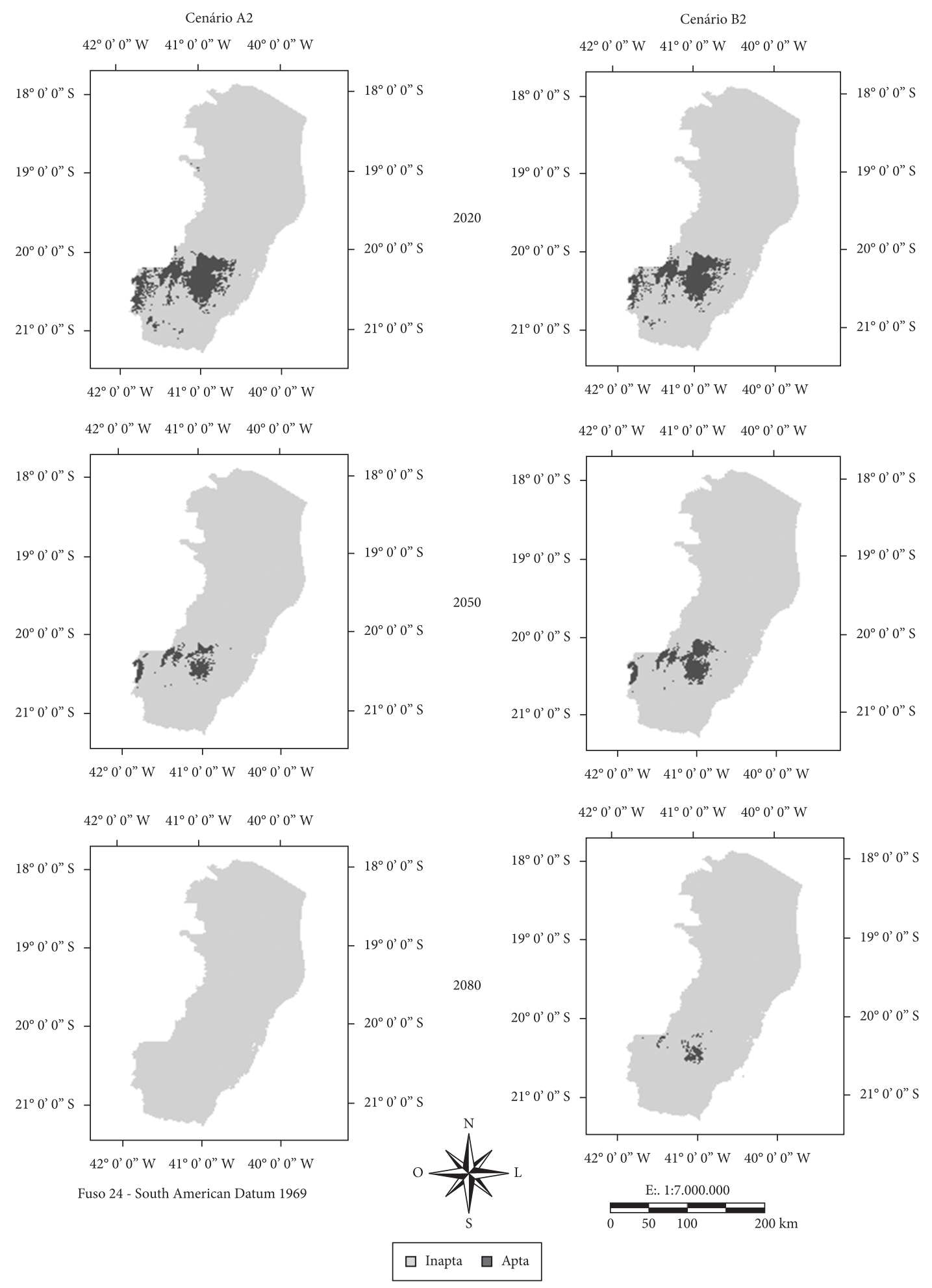

Figura 5. Zoneamento agroclimático para a espécie Pinus oocarpa nas décadas de 2020, 2050 e 2080, com seus respectivos cenários.

Figure 5. Agroclimatic zoning for Pinus oocarpa in the decades of 2020, 2050 and 2080, with their respective scenarios. 


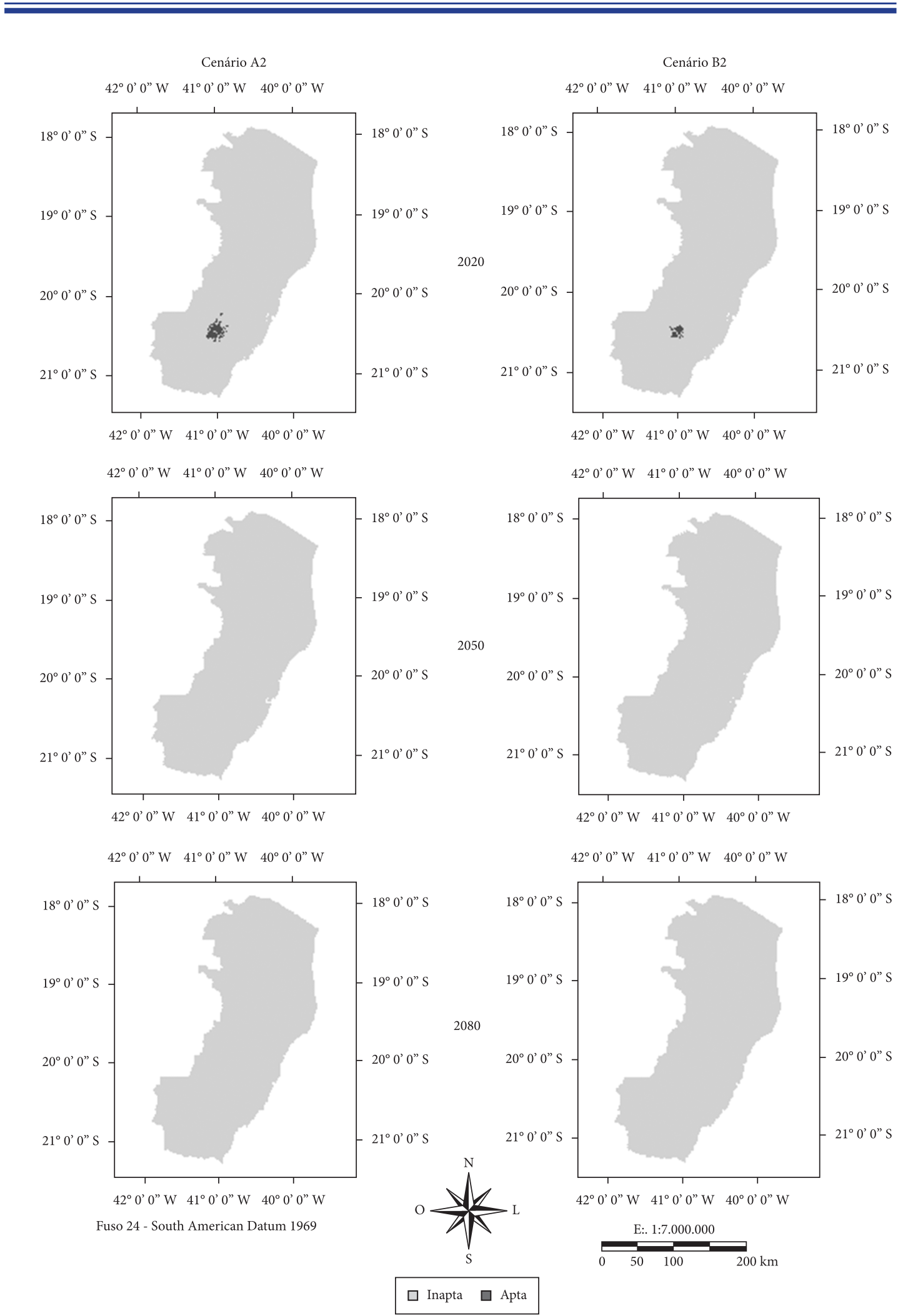

Figura 6. Zoneamento agroclimático para a espécie Pinus taeda nas décadas de 2020, 2050 e 2080, com seus respectivos cenários.

Figure 6. Agroclimatic zoning for Pinus taeda in the decades of 2020, 2050 and 2080, with their respective scenarios. 
temperatura. Observa-se que, a partir da década de 2050 e nos dois cenários analisados, passam a inexistir no Espírito Santo áreas aptas ao cultivo das espécies Pinus elliottii var. elliotti e Pinus taeda. E ainda, as áreas inaptas para o cultivo dessas espécies aumentam significativamente, chegando a $100 \%$ para todas elas, na década de 2080, no cenário A2.

As tendências encontradas foram: as áreas com melhor aptidão climática ficarão concentradas na região sul do Estado, mais precisamente nas áreas com altitudes mais elevadas e temperaturas amenas, distantes do litoral, ocasionando a interiorização da implantação da atividade silvicultural do Pinus ssp.

Convém ressaltar que este estudo não consiste em um parecer definitivo com respeito à implantação do Pinus ssp., pois as práticas de manejo silvicultural, o melhoramento genético e a utilização de clones mais resistentes ao calor podem consistir em estratégias a serem adotadas pra contornar os possíveis impactos negativos que o aquecimento global causará na produção florestal. Também, não se deve ignorar o papel da educação ambiental como ferramenta de conscientização sobre aspectos das mudanças climáticas globais e suas consequências, a fim de serem tomadas medidas para a redução drástica da emissão de gases de efeito estufa, minimizando a magnitude do aquecimento do sistema atmosférico.

Os mapas gerados definem as áreas com potenciais econômicos para a introdução das espécies em uma determinada região geográfica, servindo como base para pequenos, médios e grandes produtores que queiram investir na cultura do Pinus, podendo aumentar assim a eficiência e os lucros dessa atividade.

\section{CONCLUSÕES}

De acordo com a análise dos dados e a interpretação dos resultados obtidos dentro das condições específicas do presente trabalho, concluise que:

- O Estado do Espírito Santo apresenta áreas com aptidão climática para todas as espécies de Pinus de acordo com as bases estabelecidas para este zoneamento, no cenário atual.

- Pelo fato das espécies de Pinus elliottii var. elliottii, Pinus oocarpa e Pinus taeda apresentarem limitações climáticas bastante restritas e incompatíveis com a maioria dos índices climáticos do Estado, as áreas consideradas impróprias para a sua implantação são superiores às áreas consideradas aptas.

- Para a espécie Pinus caribaea var. hondurensis, as áreas com aptidão para implantação no Estado são mais abrangentes que as inaptas. As áreas aptas compreendem principalmente grande parte do norte do Estado e locais próximo ao litoral, onde a deficiência hídrica e as temperaturas do ar são bem mais elevadas.

- A espécie Pinus taeda apresenta áreas aptas reduzidas para sua implantação no Estado, por suas limitações climáticas serem restritas. A espécie Pinus elliottii var. elliottii apresenta comportamento semelhante a esta.

- As projeções futuras mostram a tendência ao drástico aumento de áreas inaptas ao cultivo do Pinus ssp.

- O impacto das mudanças climáticas exerce forte impacto sobre a aptidão climática das espécies Pinus elliottii var. elliottii, Pinus caribaea var. hondurensis, Pinus oocarpa e Pinus taeda, alterando sua produtividade e técnicas de manejo.

\section{STATUS DA SUBMISSÃO}

Recebido: 01/12/2010

Aceito: 01/03/2011

Reumo publicado online: 05/03/2011

Artigo completo publicado: 31/03/2011

AUTOR(ES) PARA CORRESPONDÊNCIA

\section{Laís Thomazini Oliveira}

Departamento de Engenharia Florestal, Universidade Federal do Espirito Santo - UFES, Alto Universitário, s/n, CP 16, Guararema, CEP 29500-000, Alegre, ES, Brasil e-mail: lais.thomazini@gmail.com

\section{REFERÊNCIAS}

Alfonsi RR, Pinto HS, Zullo Júnior J, Coral G, Assad ED, Evangelista BA, et al. Zoneamento climático da cultura do café (Coffea arabica) no estado do Rio de Janeiro. [cited 2007 jun. 25]. Available from: http://www.cpa. unicamp.br/cafe/index.shtml-relat_rj.html.

Assad ED, Pinto HS, Zullo Junior J, Avila AMH. Impacto das mudanças climáticas no zoneamento agroclimático do café no Brasil. Pesquisa Agropecuária Brasileira 2004; 
39(11):1057-1064. http://dx.doi.org/10.1590/S0100204X2004001100001

Bull G, Mabee W, Scharpenberg R. Global fibre supply model. Rome: Food and Agriculture Organization of the United Nations; 1998.

Carpanezzi AA, Ferreira CA, Rotta E, Namikawa IS, Sturion JA, Pereira JCD et al. Zoneamento ecológico para plantios florestais no estado do Paraná. Brasília: EMBRAPA - Centro Nacional de Pesquisa de Florestas; 1986.

Castro FS, Pezzopane JEM, Cecílio RA, Pezzopane JRM, Xavier AC. Avaliação do desempenho dos diferentes métodos de interpoladores para parâmetros do balanço hídrico climatológico. Revista Brasileira de Engenharia Agrícola e Ambiental, 2010a; 14(7): 871-880.

Castro FS, Pezzopane JEM, Cecílio RA, Pezzopane JRM, Xavier AC. Zoneamento agroclimático para espécies do gênero Pinus no estado do Espírito Santo. Floresta 2010b; 40:235-250.

Castro FS. Zoneamento agroclimático para a cultura do Pinus no estado do Espírito Santo [dissertação]. Vitória: Universidade Federal do Espírito Santo; 2008.

Cecílio RA, Medeiros SS, Silva Júnior JLC, Souza JA. Zoneamento agroclimático para a heveicultura na parte leste do Estado da Bahia. Bahia Agrícola, 2006; 7(1):14-17.

Empresa Brasileira de Pesquisa Agropecuária EMBRAPA. Sistema de produção [cited 2009 nov. 25]. Available from: http://sistemasdeproducao.cnptia. embrapa.br/Fontes HTML/Pinus/CultivodoPinus/ apresentacao.htm.

Environmental Protection Agency - EPA. The potential effects of global climate change on the Unided States. Washington: EPA; 1989. chap. 6. Agriculture. p. 93-121. EPA-230-05-89-050.

Golfari L, Caser RL, Moura VPG. Zoneamento ecológico esquemático para reflorestamento no Brasil. $2^{\mathrm{a}}$ aproximação. Belo Horizonte: Centro de Pesquisa Florestal da Região do Cerrado; 1978. PRODEPEF, Série técnica, 11.
Intergovernmental Panel on Climate Change - IPCC. Climate change 2007: the physical science basis: summary for policymakers. Geneva: IPCC; 2007.

Medeiros SS, Cecílio RA, Melo Júnior JCF, Silva Júnior JLC. Estimativa e espacialização das temperaturas do ar mínimas, médias e máximas na Região Nordeste do Brasil. Revista Brasileira de Engenharia Agrícola e Ambiental 2005; 9(3):247-255. http://dx.doi. org/10.1590/S1415-43662005000200016

Revista da Madeira - REMADE [cited 2009 jan. 26]. 2002; 12. Available from: http://www.remade.com.br/ pt/revista_capa.php?edicao $=68$.

Revista da Madeira - REMADE [cited 2010 nov. 25] 2008; 115. Available from: http://www. remade.com.br/br/revistadamadeira_materia. php? num $=1300 \&$ subject $=E \% 20$ mais \& title $=\mathrm{A} \% 20$ cadeia $\% 20$ produtiva $\% 20$ do $\% 20$ Pinus $\% 20$ no $\% 20$ RS\%20situa\%E7\%E3o\%20atual,\%20a\%E7\%F5es\%20 e\%20perspectivas.

Sediyama GC, Melo Júnior JCF. Modelos para estimativas das temperaturas normais mensais médias, máximas, mínimas e anual no estado de Minas Gerais. Engenharia na Agricultura 1998; 6(1):57-61.

Siqueira JDP, Lisboa RS, Ferreira AM, Souza MFR, Araújo E, Lisbão Júnior L et al. Estudo ambiental para os programas de fomento florestal da Aracruz Celulose S. A. e extensão florestal do governo do estado do Espírito Santo. Revista Floresta 2004; 34:03-67.

Siqueira OJF, Farias JRB, Sans LM. Potential effects of global climate change for brazilians agriculture and adaptative strategies for wheat, maize and soybean. Revista Brasileira de Agrometeorologia 1994; 2:115-129.

Thornthwaite CW, Mather JC. The water balance. Centeron: Drexel Institute of Technology, Laboratory of Climatology; 1955. Publications in climatology, 8(1).

Thornthwaite CW. An approach toward a rational classification of climate. Geographical Review 1948; 38:55-94. http://dx.doi.org/10.2307/210739 